\title{
The changing epidemiology of invasive aspergillosis in the non-traditional host: Risk factors and outcomes
}

\author{
Jose A Vazquez ${ }^{1}$, Maria Paula Tovar-Torres ${ }^{2}$, Ameet Hingwe ${ }^{1}$, Faiqa Cheema ${ }^{1}$, Verna L Welch ${ }^{3}$ and Kimbal D Ford ${ }^{3}$ \\ ${ }^{1}$ Division of Infectious Diseases, Medical College of Georgia/Augusta University, Augusta, GA, USA \\ ${ }^{2}$ Division of Infectious Disease, Henry Ford Hospital, Detroit, MI, USA \\ ${ }^{3}$ Pfizer Inc., Collegeville, PA, USA
}

\begin{abstract}
Background: Invasive fungal infections due to Aspergillus spp. are common in 'high-risk' immunocompromised patients. Recently, invasive aspergillosis (IA) in the 'low-risk' or 'non-traditional' host has been increasingly recognized. Unfortunately, risk factors and diagnostic modalities for this infection are poorly described. This study's goal is to further characterize IA in the population classically described as 'low-risk' and infrequently studied.

Methods: A retrospective cohort of patients with proven or probable IA (excluding those with neutropenia or hematologic malignancy) was evaluated.

Results: Thirty-six patients with either proven (23) or probable (13) IA met inclusion criteria. Mean age was 54.6 years. The most common risk factors identified in patients with IA were steroid use (69\%), ICU stay (56\%), immunosuppressive agents (39\%), solid organ transplant (33\%), diabetes (28\%) and mechanical ventilation (28\%). The most common sites of infection were lungs (67\%) and sinuses (19\%). A. fumigatus was the most prevalent spp. isolated (63\%), followed by A. niger (16\%). Additionally, 17 patients had bacterial coinfections. Voriconazole was the first-line antifungal used in 23 patients, followed by combination antifungal therapy in eight patients. Clinical success was noted in $72 \%$ of patients with a $25 \%$ cumulative mortality 90 days after diagnosis.

Conclusions: This study describes IA in a population infrequently studied and considered to be at low risk when compared to the neutropenic population of patients. Awareness of risk factors in this 'non-traditional' host, improved diagnostic techniques, and earlier initiation of appropriate antifungal therapy should improve overall survival and response to therapy. Larger, multicenter epidemiologic studies in similar populations are warranted to improve the understanding of this underestimated infection.
\end{abstract}

\section{Introduction}

Over the last two decades, the incidence of invasive fungal disease has risen, due in part to an increase in immunocompromised patients, resulting as a consequence of advances in transplantation, chemotherapy, use of monoclonal antibodies and the increase in complex invasive surgical procedures [1]. Invasive fungal infections due to the mould Aspergillus, have been well described and evaluated in patients who are known to be at high risk, specifically in patients with underlying hematologic malignancies, neutropenia or in recipients of solid organ or hematopoietic stem cell transplant (HSCT) [2]. The comparative distribution of invasive mould infections varies among the type of organ transplanted [3-5]. Overall, invasive aspergillosis (IA) and other mold infections predominated among HSCT recipients [3-5]. Among the solid organ transplant recipients, IA was most common in lung transplant recipients accounting for $44 \%$ of all IFI, compared to $23 \%, 14 \%, 11 \%, 5 \%$ in heart, kidney, liver and pancreas transplant recipients, respectively $[4,5]$. The estimated occurrence of IA is $5 \%-13 \%$ in HSCT recipients and $10 \%-20 \%$ in patients receiving intensive chemotherapy for leukemia $[3,6]$. Invasive aspergillosis is a severe, progressive infection that is associated with high morbidity and mortality $(30 \%-85 \%)$ in these 'high-risk' or 'classic' patients. However, survival can reach $50 \%$ in patients that are diagnosed early and in those where appropriate and early antifungal therapy is initiated [3-6].

Recent clinical studies have shown that IA appears to be an underestimated opportunistic fungal infection in patients that are not known to be either critically ill or severely immunosuppressed $[7,8]$. In the past decade, infections due to Aspergillus spp. have been increasingly identified in patients who were not traditionally considered to be at risk for aspergillosis [7,9-17]. These 'low-risk' or 'non-traditional' patients with IA have included patients with underlying chronic obstructive pulmonary disease (COPD), liver failure, renal failure, diabetes mellitus, near-drownings and intensive care unit (ICU) patients [6-11].

The true incidence of IA in these 'low-risk' or 'non-traditional' hosts is frequently underestimated due to a low index of suspicion, a lack of consistent case definitions, absence of surveillance measures and a low sensitivity of current diagnostic assays [7-9]. The goal of this retrospective cohort study is to better describe the characteristics, risk factors and outcomes of these 'low-risk' or 'non-traditional' patients with a diagnosis of IA.

Correspondence to: Jose A Vazquez MD, Division of Infectious Diseases, Medical College of Georgia/Augusta University, 1120 15th Street, Room 3029, Augusta, GA 30912, USA, Tel: +1 706-721-1244; Fax: +1 706-721-4517; E-mail: jvazquez@augusta.edu

Key words: epidemiology, invasive aspergillosis, 'low-risk/non-traditional' host, voriconazole, Aspergillus sp

Received: June 02, 2016; Accepted: August 05, 2016; Published: August 09, 2016 


\section{Methods}

\section{Study design}

Patients and methodology: A retrospective evaluation of a cohort of eligible patients was created using the electronic medical records (EMR) from the Henry Ford Health System (HFHS), Detroit, Michigan, USA over a 4-year period (2007-2010). Henry Ford Hospital (HFH) is a tertiary-care teaching hospital with 900 beds. Forty patients with a diagnosis of IA were initially identified; 36 patients met the inclusion criteria and were included in the analysis. The study was approved by the Institutional Review Board of Henry Ford Hospital. Data was extracted from HFHS EMR records by two of the authors and verified by a data adjudication committee.

Cases of IA defined as proven or probable according to the revised European Organisation for Research and Treatment of Cancer/Mycoses Study Group (EORTC/MSG) criteria were eligible for inclusion [18].

Patients with a history of neutropenia, hematologic malignancy, bone marrow transplant, aplastic anemia, myelodysplastic syndrome, and allergic bronchopulmonary aspergillosis and allergic fungal sinusitis were excluded from the study.

Patient classification: In this study, proven IA was defined as a clinically compatible illness with one or more of the following: Aspergillus spp. isolated from a normally sterile site; a biopsy specimen or aspirate with hyphae consistent with the presence of Aspergillus spp., and positive culture of Aspergillus spp. from the same organ [18].

The presence of host factors, clinical features and mycological evidence were required for a probable IA classification [18]. Probable IA in this study was defined as a clinically compatible illness plus one or more of the following: a positive culture of Aspergillus spp. from bronchoalveolar lavage (BAL) fluid, sputum or aspirate; hyphae consistent with the presence of Aspergillus spp. in a biopsy specimen, BAL fluid, sputum or aspirate; galactomannan antigen detected in plasma, serum, BAL fluid or cerebrospinal fluid; $\beta$-D-glucan detected in serum.

Evaluations: The patient characteristics extracted from the HFHS EMR records included patient demographics, prior medical history, and prior medications including antimicrobials, antifungals and any immune suppressive agent. Diagnostic evaluations included tissue cultures, BAL, histopathology, serologic assays, blood cultures, microbiology, and radiologic studies. Pharmacy records, hospitalization services, length of stay, outpatient services and insurance information were captured. The results of autopsies, when performed, were also evaluated for this cohort.

In addition, minimum inhibitory concentrations were performed on all Aspergillus spp. isolates that were available using the Clinical Laboratory Standard Institute criteria [19].

Clinical outcome: Clinical response was evaluated at the time of discharge, end of antifungal therapy, 90 days and six months after the diagnosis of IA. In addition, survival was also evaluated at the time of discharge, at the end of therapy, at 90 days, 6 months, and 12 months after the initial diagnosis of IA.

\section{Statistical analyses}

Descriptive statistics including mean, median, range, or frequency and percentage were used to describe the data evaluated in this study. The risk factor prevalence was calculated as the frequency of the risk factor among the study sample. No formal statistical evaluations were performed.

\section{Results}

\section{Demographic and clinical characteristics}

During the 4-year period, 40 patients with a diagnosis of IA were initially identified. However, only 36 of these patients met the study inclusion criteria and were included in the analysis. This included 23 patients with proven IA and 13 with probable IA. Slightly more than half of the patients evaluated were male and the mean age was 54.6 years (Table 1). Patients with proven IA had lower Acute Physiology and Chronic Health Evaluation (APACHE) II scores than probable IA patients (mean [standard deviation] 9.78 [6.8] and 14.25 [5.1], respectively).

\section{Prevalence of risk factors and underlying disease}

The most common risk factors identified in patients with the diagnosis of IA in this study were steroid use (69\%), ICU stay (56\%), immunosuppressive agent use (39\%), solid organ transplant (33\%), diabetes mellitus (28\%), and mechanical ventilation (28\%) (Figure 1). There were few differences between proven and probable IA (Table 2). Eleven patients received a solid organ transplant, five had a kidney transplant, four underwent a liver transplant and two had lung transplants. Twenty-five patients received corticosteroids including 7 (21\%) prescribed multiple formulations. Of these, 20 (80\%) received oral therapy, $6(24 \%)$ used nasal inhalation and $8(32 \%)$ were on oral

Table 1. Demographic and clinical characteristics of patients with proven and probable invasive aspergillosis.

\begin{tabular}{|c|c|c|c|}
\hline Characteristic & $\begin{array}{c}\text { Total } \\
\mathbf{n}=\mathbf{3 6}\end{array}$ & $\begin{array}{c}\text { Proven } \\
\mathbf{n}=\mathbf{2 3}\end{array}$ & $\begin{array}{c}\text { Probable } \\
n=13\end{array}$ \\
\hline Age, years, mean (SD) & $54.6(13.5)$ & $51.5(13.7)$ & $60.0(11.7)$ \\
\hline Male gender, n (\%) & $21(58.4)$ & $14(60.9)$ & $7(53.9)$ \\
\hline $\begin{array}{c}\text { Race, } \mathrm{n}(\%) \\
\text { White } \\
\text { Black } \\
\text { Other }\end{array}$ & $\begin{array}{c}18(50.0) \\
14(38.9) \\
4(11.1)\end{array}$ & $\begin{array}{c}9(39.1) \\
12(52.2) \\
2(8.7)\end{array}$ & $\begin{array}{l}9(69.2) \\
2(15.4) \\
2(15.4)\end{array}$ \\
\hline $\begin{array}{l}\text { Insurance, } \mathrm{n}(\%) \\
\text { Health maintenance organization } \\
\text { Commercial } \\
\text { Medicaid } \\
\text { Medicare } \\
\text { No insurance }\end{array}$ & $\begin{array}{l}18(50.0) \\
2(5.6) \\
2(36.1) \\
13(2.8) \\
1\end{array}$ & $\begin{array}{c}13(56.5) \\
2(8.7) \\
1(4.4) \\
6(26.1) \\
1(4.4)\end{array}$ & $\begin{array}{c}5(38.5) \\
0 \\
1(7.7) \\
7(53.9) \\
0\end{array}$ \\
\hline APACHE II score, mean (SD) & $11.4(6.5)$ & $9.8(6.8)$ & $14.2(5.1)$ \\
\hline Comorbidities, mean (SD) & $3.9(3.2)$ & $3.5(2.8)$ & $4.5(3.8)$ \\
\hline
\end{tabular}

Table 2. Risk factors and underlying disease in patients with proven and probable invasive aspergillosis.

\begin{tabular}{|l|c|c|c|}
\hline Risk factor/underlying disease & $\begin{array}{c}\text { Total } \\
\mathbf{n = 3 6}\end{array}$ & $\begin{array}{c}\text { Proven } \\
\mathbf{n = 2 3}\end{array}$ & $\begin{array}{c}\text { Probable } \\
\mathbf{n = 1 3}\end{array}$ \\
\hline Steroids & 25 & 20 & 5 \\
\hline ICU stay & 20 & 13 & 7 \\
\hline Immunosuppression & 19 & 12 & 7 \\
\hline Solid organ transplant & 11 & 4 & 7 \\
\hline Diabetes mellitus & 10 & 6 & 4 \\
\hline Mechanical ventilation & 10 & 7 & 3 \\
\hline COPD & 8 & 5 & 3 \\
\hline Sarcoidosis & 8 & 4 & 4 \\
\hline Malignancy & 8 & 5 & 3 \\
\hline Collagen vascular disease & 6 & 4 & 2 \\
\hline Alcohol abuse & 4 & 4 & 0 \\
\hline Cirrhosis & 3 & 2 & 1 \\
\hline
\end{tabular}




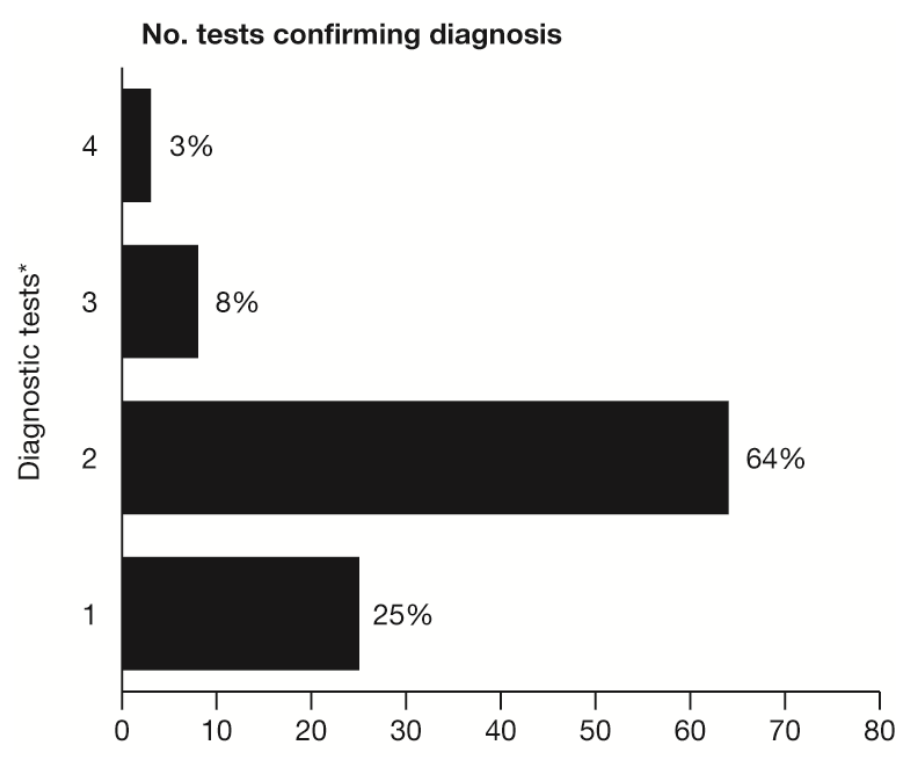

Patients with positive test results (\%)

${ }^{*} \mathrm{CT}$ scan, culture (for example BAL, sputum), tissue biopsy and galactomannan

Figure 1. The number of diagnostic testing performed to confirm the diagnosis of proven or probable invasive aspergillosis.
Aspergillus fumigatus Aspergillus flavus
Aspergillus niger

Aspergillus glaucus

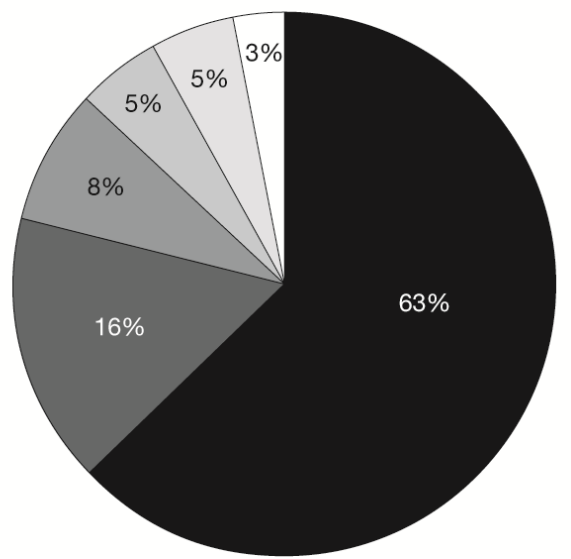

Other Aspergillus spp. Aspergillus terreus
Figure 2. Distribution of Aspergillus spp. isolates recovered from clinical specimens.

inhalation. The most frequently used immunosuppressive agents were tacrolimus (30.6\%), mycophenolate mofetil (19.4\%), sirolimus (11.1\%) and methotrexate (8.3\%).

\section{Diagnostic evaluations}

Patients frequently underwent numerous diagnostic modalities to establish the diagnosis of IA. These included radiological testing, biopsy, serological assays and microbiological assays (Figure 2). Aspergillosis was confirmed in the majority of patients who met inclusion criteria $(23 / 36,64 \%)$. A total of $69 \%$ of patients had a CT scan that supported the diagnosis. The same number of patients also had positive fungal cultures $(24 / 36,69 \%)$, whereas, $42 \%(15 / 36)$ of patients had a tissue biopsy diagnostic for aspergillosis. Galactomannan assays were not routinely ordered, however 34 galactomannan assays were performed in 19 patients, but only three were positive.

Multiple sites of infection were observed, with the lungs (67\%) being the most predominant location followed by the sinuses (19\%), brain (8\%) and cutaneous sites (3\%). In patients with proven infection, $35 \%$ had pulmonary infections, $30 \%$ had sinus infections, $17 \%$ had intraabdominal infections, $9 \%$ had a CNS infection, $4 \%$ had fungemia, and $4 \%$ had endocarditis. In contrast, in patients stratified into probable IA infections, $77 \%$ had a pulmonary infection, and $23 \%$ had a sinus infection, there were no patients with CNS or disseminated infection in this group. Three patients (8\%) had evidence of disseminated IA, as defined by more than one site of infection. Furthermore, A. fumigatus was the most commonly isolated species (63\%), followed by A. niger in $16 \%$ of patients (Figure 3 ).

Interestingly enough, bacterial co-infections were commonly observed in patients with IA and were found in 17 patients (50\%). The majority, (12) were seen in patients with proven IA and five in patients with probable IA. The most frequently isolated bacteria included Streptococcus spp. and gram-negative bacilli (Pseudomonas aeruginosa and Haemophilus spp.) recovered from $32 \%$ and $24 \%$ of patients, respectively.

In vitro susceptibility testing was performed on 22 Aspergillus spp. isolates that were recovered from patients. All isolates were found to be susceptible to all of the antifungals tested, except for one Aspergillus isolate that developed secondary resistance to voriconazole, but remained susceptible to posaconazole. This A. flavus isolate was recovered from a lung transplant recipient who developed pulmonary aspergillosis and Aspergillus empyema and subsequently required greater than 6 months of voriconazole therapy. Although the initial Aspergillus isolates were susceptible to voriconazole and posaconazole, the final Aspergillus isolate recovered just prior to the patient's demise was found to be voriconazole-resistant yet remained susceptible to posaconazole.

\section{Antifungal therapy}

Thirty-four of the 36 patients received antifungal therapy for IA, with $68 \%(23 / 34)$ of the patients receiving voriconazole monotherapy as the first-line antifungal agent. In addition, combination antifungal therapy was used in 8 patients; 6 patients received voriconazole and

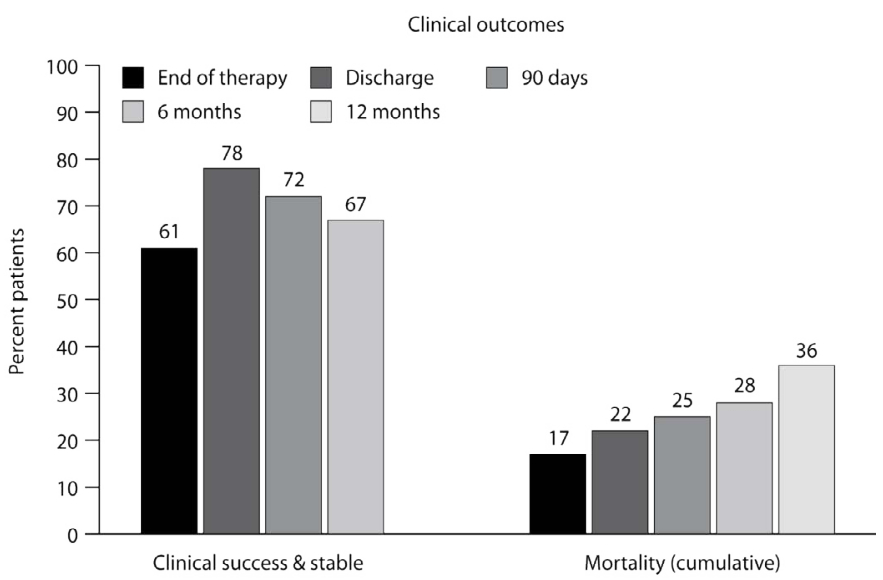

Figure 3. Clinical outcomes in patients with proven or probable invasive aspergillosis. 
anidulafungin, 1 patient received lipid amphotericin $B$ and posaconazole and 1 patient received lipid amphotericin $B$ and flucytosine. Two patients were not treated with any form of antifungal therapy and they both died within the first 30 days. Overall for the entire group, the mean duration of antifungal therapy was 201 days (Table 3).

\section{Resource utilization}

The majority of patients $(32 / 36,88.9 \%)$ had at least one inpatient hospital stay, with a mean length of stay of 66.6 days. Over half (55.6\%) of the patients were admitted to the ICU at least once during their hospitalization (Table 3).

\section{Clinical outcome}

The overall clinical success rate was noted to be $72 \%$ within 90 days after the end of antifungal therapy, with a cumulative all-cause mortality rate of $25 \%$ (Figure 4). It is important to note that one-third $(4 / 12)$ of deaths were attributable to the fungal infection. No differences in clinical success or mortality were noted between the proven IA or probable IA patient groups.

\section{Discussion}

This retrospective cohort study describes IA in a population of patients considered to be at low risk and in some cases at no risk of infection due to Aspergillus spp. In this patient cohort, steroid use was the most common risk factor associated with the development and subsequent diagnosis of IA. The use of steroids as a risk factor in 'non-traditional' patients has been previously noted by Meersseman et al. [6]. Other important risk factors identified in this study were chronic conditions such as diabetes mellitus, COPD, sarcoidosis, those receiving immune suppressants and those on mechanical ventilation.

As previously described in HSCT recipients, two-thirds of the patients with a diagnosis of IA had infections that involved the lungs, followed by the sinuses (19\%). Central nervous system infections and disseminated infections were also seen in $8 \%$ of cases. As previously described in HSCT recipients, most infections were due to A. fumigatus followed by $A$. niger. In contrast, most epidemiologic studies of IA with positive cultures report A. fumigatus as the most frequently isolated Aspergillus spp. followed by A. flavus [2-6].

The diagnosis of IA remains difficult in any patient population. However, in this so called 'low-risk' or intermediate risk population, due to the lack of epidemiologic data and poor and non-specific diagnostic techniques, the diagnosis is frequently missed or not even considered. Consequently, the diagnosis is often not made while the patient is alive or is made too late when the infection is advanced. Furthermore, because the current EORTC/MSG criteria were not evaluated nor

Table 3. Resource utilization for patients with proven and probable invasive aspergillosis.

\begin{tabular}{|l|c|}
\hline Utilization category & $\mathrm{n}(\%)$ \\
\hline Patients having hospital stay, $\mathrm{n}(\%)$ & $32(88.9)$ \\
\hline $\begin{array}{l}\text { Hospital length of stay, days } \\
\text { Mean (SD) } \\
\text { Median [IQR] }\end{array}$ & $66.6(169.4)$ \\
\hline ICU stay, n (\%) & $18[10.5,38.5]$ \\
\hline $\begin{array}{l}\text { ICU length of stay, days } \\
\text { Mean (SD) } \\
\text { Median [IQR] }\end{array}$ & $20(55.6)$ \\
\hline $\begin{array}{l}\text { Days of antifungal therapy } \\
\text { Mean (SD) } \\
\quad \text { Median [IQR] }\end{array}$ & $21.4(32.6)$ \\
\hline ICU, intensive care unit; IQR, inter-quartile range; SD, standard deviation. \\
\hline
\end{tabular}

established in this unique patient population, the gold standard of diagnosis remains the tissue biopsy. Unfortunately, if an invasive procedure to obtain a tissue biopsy is not performed, the diagnosis is typically based on a combination of clinical characteristics, underlying disease state, risk factors, radiologic manifestations, microbiology and serologic assays $[2,13,18,20]$. Criteria for the diagnosis of IA in patients who are considered to be high-risk have recently been updated and new diagnostic tools such as serum galactomannan have been approved $[18,20]$. However, these revised criteria are not necessarily compatible with establishing the diagnosis of IA in this 'non-traditional' patients and still need to be fully evaluated by investigators in 'low-risk' hosts before they are considered standard of care $[18,20]$.

The diagnosis of IA in 'non-traditional' patients is further hampered by various factors including difficulty in interpreting complex clinical manifestations in complicated patients who may be on mechanical ventilation and may also simultaneously have underlying hospital acquired or ventilator-acquired bacterial pneumonia or a prior history of underlying chronic lung disease (COPD, sarcoidosis), which frequently interferes with the radiological characteristics and subsequent diagnosis of IA [8-11]. Furthermore, the conventional tests and serologic assays (galactomannan and $\beta$-D-glucan) that are generally used to diagnose IA in known high-risk host patients have not been fully evaluated or validated in the 'low-risk' host. Recent data suggest that these diagnostic assays are not as sensitive or specific as they are in the 'classic' patients with IA such as neutropenics or HSCT recipients $[8-11,18,20]$. In the 'classic' patient, CT scans and serum galactomannan assays are generally considered sensitive and specific in establishing the diagnosis of IA $[18,20]$. In contrast, in the 'lowrisk' host, these tests have sensitivities of less than $50 \%$, and thus are frequently negative and not helpful in establishing an early diagnosis of IA [8-10]. In addition, microbiological diagnosis of respiratory specimen cultures are also hindered by the low sensitivity and lack of specificity that is dependent upon the patients immunocompromised status [8-10]. It is likely that those patients who received steroids in this study have a significant degree of immunosuppression, possibly as severe as acute leukemia patients or HSCT recipients. Therefore, the finding of a positive Aspergillus culture from the BAL fluid should be equally as supportive of a diagnosis of IA. Furthermore, the clinical response to antifungal therapy observed in this study suggests that these patients did indeed have an invasive fungal infection.

This study demonstrates that due to the difficulties in establishing an early and accurate diagnosis of IA, these patients frequently underwent multiple diagnostic modalities. The majority of patients required at least two positive diagnostic tests in order to confirm the diagnosis of IA. Although not as specific or sensitive as in HSCT recipients, the CT scan results frequently were able to corroborate the diagnosis in $69 \%$ of patients. An important finding was the poor sensitivity of serum galactomannan determinations in this patient population. In fact, only three (9\%) of the galactomannan assays were positive. This is in contrast to the $70 \%-90 \%$ sensitivity described in either neutropenic patients or HSCT recipients [6,7]. This further demonstrates the difficulties in establishing a diagnosis of IA in these 'low-risk' patients.

In this study voriconazole, which is the recommended and standard treatment for most forms of IA was the most commonly used antifungal in this cohort of patients despite the lack of clinical studies in this specific patient population [21]. Interestingly, the second most common form of antifungal therapy used was combination therapy (anidulafungin and voriconazole), again despite the lack of clinical studies. 
The overall mortality rate reported in this cohort was 25\% (90 days after diagnosis), which is significantly lower than the reported mortality rates in patients with underlying hematologic malignancies or HSCT recipients (50\%-85\%) [2-11], but underlies the importance of IA in these unique patients considered to be at low risk. The data presented here in the 'non-traditional' or 'low-risk' patients with IA differs from that observed with 'classic' patients who are at high risk of IA, such as those who are neutropenic or recipients of HSCT. This difference in mortality probably relates to the severity of underlying disease, severity of immunosuppression and the multiple co morbidity factors that are typical for the 'high-risk' patients, when compared to the 'low-risk' patients identified in this study [3-5, 8-11].

\section{Conclusion}

In conclusion, the objective of this study was to increase the understanding and awareness of IA in this 'low-risk' and poorly studied population and to better defines the plausible risk factors associated with IA in these 'non-traditional' patients. This new knowledge will lead to an increased awareness of this poorly studied and poorly described infectious entity and thus elicit an increased index of suspicion, an aggressive search for the correct diagnosis, an earlier establishment of the diagnosis and subsequently the early initiation of appropriate antifungal therapy. All of these factors together may ultimately influence the overall morbidity, response to antifungal therapy and survival. As would be expected in this type of descriptive analysis, there are several limitations to this study. Specifically, the study is a small, single-center, retrospective study without controls. Moreover, because it is a retrospective study, data collection for all patients was not standardized, nor available. Certainly, larger, prospective, multicenter epidemiologic studies in similar populations are warranted to further expand and improve the understanding of this deadly and greatly underestimated fungal infection.

\section{Acknowledgments}

This study was sponsored in part by a research grant from Pfizer Inc. We would like to thank Roger Luo, PhD, of Pfizer Inc., for his assistance with study database design. Editorial support was provided by Fiona Boswell, $\mathrm{PhD}$, at Complete Medical Communications and funded by Pfizer Inc.

\section{Conflict of interest}

KDF is an employee of Pfizer Inc. VLW was an employee of Pfizer during the time the study was conducted. HFH received support from Pfizer for JAV to travel to a meeting. All other authors have no conflict of interest.

\section{References}

1. Morgan J (2005) Global trends in candidemia: review of reports from 1995-2005. Curr Infect Dis Rep 7: 429-439. [Crossref]

2. [No authors listed] (1997) Guidelines for prevention of nosocomial pneumonia. Centers for Disease Control and Prevention. MMWR Recomm Rep 46: 1-79. [Crossref]

3. Kontoyiannis DP, Marr KA, Park BJ, Alexander BD, Anaissie EJ, et al. (2010)
Prospective surveillance for invasive fungal infections in hematopoietic stem cell transplant recipients, 2001-2006: overview of the Transplant-Associated Infection Surveillance Network (TRANSNET) Database. Clin Infect Dis 50: 1091-00.

4. Neofytos D, Fishman JA, Horn D, Anaissie E, Chang CH, et al. (2010) Epidemiology and outcome of invasive fungal infections in solid organ transplant recipients. Transpl Infect Dis 12: 220-29.

5. Pappas PG, Alexander BD, Andes DR, Hadley S, Kauffman CA, et al. (2010) Invasive fungal infections among organ transplant recipients: results of the TransplantAssociated Infection Surveillance Network (TRANSNET). Clin Infect Dis 50: 11011111. [Crossref]

6. Harman EM. Aspergillosis.

7. Trof RJ, Beishuizen A, Debets-Ossenkopp YJ, Girbes AR, Groeneveld AB (2007) Management of invasive pulmonary aspergillosis in non-neutropenic critically ill patients. Intensive Care Med 33: 1694-1703. [Crossref]

8. Meersseman W, Lagrou K, Maertens J, Van Wijngaerden E (2007) Invasive aspergillosis in the intensive care unit. Clin Infect Dis 45: 205-216. [Crossref]

9. Meersseman W, Vandecasteele SJ, Wilmer A, Verbeken E, Peetermans WE, et al. (2004) Invasive aspergillosis in critically ill patients without malignancy. Am J Respir Crit Care Med 170: 621-625. [Crossref]

10. Rello J, Esandi ME, Mariscal D, Gallego M, Domingo C, et al. (1998) Invasive pulmonary aspergillosis in patients with chronic obstructive pulmonary disease: report of eight cases and review. Clin Infect Dis 26: 1473-75.

11. Cornet M, Mallat H, Somme D, Guérot E, Kac G, et al. (2003) Fulminant invasive pulmonary aspergillosis in immunocompetent patients--a two-case report. Clin Microbiol Infect 9: 1224-1227. [Crossref]

12. Damle BD, Dowell JA, Walsky RL, Weber GL, Stogniew M, et al. (2009) In vitro and in vivo studies to characterize the clearance mechanism and potential cytochrome $\mathrm{P} 450$ interactions of anidulafungin. Antimicrob Agents Chemother 53: 1149-56.

13. Bulpa P, Dive A, Sibille Y (2007) Invasive pulmonary aspergillosis in patients with chronic obstructive pulmonary disease. Eur Respir J 30: 782-800. [Crossref]

14. Ader F, Nseir S, Le Berre R (2005) Invasive pulmonary aspergillosis in chronic obstructive pulmonary disease: an emerging fungal pathogen. Clin Microbiol Infect 11: 427-29.

15. Khasawneh F, Mohamad T, Moughrabieh MK, Lai Z, Ager J, et al. (2006) Isolation of Aspergillus in critically ill patients: a potential marker of poor outcome. J Crit Care 21: 322-327. [Crossref]

16. Samarakoon P, Soubani A (2008) Invasive pulmonary aspergillosis in patients with COPD: a report of five cases and systematic review of the literature. Chron Respir Dis 5: 19-27. [Crossref]

17. Ader F, Bienvenu AL, Rammaert B, Nseir S (2009) Management of invasive aspergillosis in patients with COPD: rational use of voriconazole. Int J Chron Obstruct Pulmon Dis 4: 279-287. [Crossref]

18. De Pauw B, Walsh TJ, Donnelly JP (2008) Revised definitions of invasive fungal disease from the European Organization for Research and Treatment of Cancer/ Invasive Fungal Infections Cooperative Group and the National Institute of Allergy and Infectious Diseases Mycoses Study Group (EORTC/MSG) Consensus Group. Clin Infect Dis 46: 1813-21.

19. Clinical and Laboratory Standards Institute (2009) M23 development of in vitro susceptibility testing criteria and quality control parameters. Clinical and Laboratory Standards Institute Document 10: 1-53.

20. Ruhnke M, Bohme A, Buchheidt D, Cornely O, Donhuijsen K, et al. (2012) Diagnosis of invasive fungal infections in hematology and oncology--guidelines from the Infectious Diseases Working Party in Haematology and Oncology of the German Society for Haematology and Oncology (AGIHO). Ann Oncol 23: 823-33.

21. Herbrecht R, Denning DW, Patterson TF, Bennett JE, Greene RE, et al. (2002) Voriconazole versus amphotericin B for primary therapy of invasive aspergillosis. $N$ Engl J Med 347: 408-415. [Crossref]

Copyright: C2016 Vazquez JA. This is an open-access article distributed under the terms of the Creative Commons Attribution License, which permits unrestricted use, distribution, and reproduction in any medium, provided the original author and source are credited. 